\title{
Invention and Pilot Study of the Efficacy and Safety of the SUPRAtube Device in Continuous Supraglottic Aspiration for Intubated and Mechanically Ventilated Patients
}

\author{
Alba Ramírez-Sarmiento (iD ${ }^{1-3}$ \\ Orlando Aya ${ }^{3,4}$ \\ Diana Cáceres-Rivera (D) ${ }^{1,2}$ \\ Carlos F Reyes $^{2-4}$ \\ Angela Espitia (iD ${ }^{1,2}$ \\ Camilo Pizarro 3,4 \\ Joaquim Gea (iD ${ }^{5,6}$ \\ Victor R Castillo 7,8 \\ Mauricio Orozco-Levi (D) $^{1-3,6}$ \\ 'Respiratory Department, Instituto \\ Cardiovascular y Hospital Internacional \\ de Colombia, Fundación Cardiovascular \\ de Colombia, Floridablanca, Santander, \\ Colombia; ${ }^{2}$ EMICON Research Group \\ MINCIENCIAS, Bogotá, Cundinamarca, \\ Colombia; ${ }^{3}$ Universidad de Santander \\ (UDES), Bucaramanga, Santander, \\ Colombia; ${ }^{4}$ Department of Critical Care, \\ Fundación Cardiovascular de Colombia, \\ Floridablanca, Santander, Colombia; \\ ${ }^{5}$ Respiratory Department, Parc de Salut \\ Mar, Barcelona, Catalonia, Spain; ${ }^{6}$ CEXS- \\ Universidad Pompeu Fabra, Barcelona, \\ Catalonia, Spain; ${ }^{7}$ Department of \\ Cardiovascular Surgery, Fundación \\ Cardiovascular de Colombia, \\ Floridablanca, Santander, Colombia; \\ ${ }^{8}$ Biomedical and Translational Research \\ Group, Fundación Cardiovascular de \\ Colombia, Floridablanca, Santander, \\ Colombia
}

Correspondence: Mauricio Orozco-Levi Respiratory Department, ICV-HIC, Fundación Cardiovascular de Colombia, Calle I55a no. 23-58, El Bosque, Floridablanca, Santander, Colombia Tel +57 317574l42।

Email mauricioorozco@fcv.org
Background: Bronchoaspiration of content that accumulates in the supraglottic area (eg, saliva, gastroesophageal reflux) is a risk factor for ventilator-associated pneumonia. A continuous supraglottic suction system may decrease the risk of bronchoaspiration in these patients.

Objective: (1) Constructing a conceptual model and functional prototype of a continuous supraglottic suction device for use in humans; (2) defining functional characteristics in ex vivo swine head models; and (3) evaluating its efficacy and safety in mechanically ventilated patients. Methods: Study conducted in three phases. First phase: definition of distances and diameters of the triangle determined by dental arch, posterior oropharynx and vallecula, and diameter of the oropharynx in axial projection; and identification of the declining area of supraglottic suction. Second phase: design engineering and functional prototype evaluated in ex vivo models. Third phase: evaluation of device use in terms of safety and efficacy in ventilated patients.

Results: We obtained a final functional model of the SUPRAtube device injected into PVC for medical use. Device effectiveness in in vitro simulation showed a high and fast suction capacity of liquid and thick volumes. Study of swine heads allowed to validate the shape, size and functional fenestration of the device. Study in intubated and mechanically ventilated patients showed a high supraglottic suction capacity and the absence of local adverse events during 72 (7-240) hours of continuous operation.

Conclusion: Our study describes the process of conceptualization, design and production of a practical, safe, low-cost continuous supraglottic suction device without representing antibiotic pressure, which appears to be a new complementary preventive strategy for the standard management of intubated and mechanically ventilated patients.

Keywords: mechanical ventilation, bronchoaspiration, supraglottic suction, SUPRAtube, endotracheal tube, gastroesophageal reflux

\section{Introduction}

The present study describes the conceptual design, functional prototyping and final deliverable of a technological innovation device designed for continuous supraglottic suction in intubated and mechanically ventilated patients, named with the acronym SUPRAtube. This device has received a patent as a utility model (ref. NC2016/0002059 Resolution 466 of the Superintendence of Industry and Commerce, Colombia) due to its novel nature, in the absence of similar devices 
on market, as a complementary solution for the prevention of complications such as nosocomial pneumonia in these patients.

Patients under invasive mechanical ventilation (MV) suffer a high incidence of gastroesophageal reflux (GER) as well as an accumulation of saliva and other secretions in the oropharyngeal area. ${ }^{1,2}$ Macroaspiration in critically ill patients witnessed by health personnel range from $1 \%$ to $12 \%$ of frequency. Microaspiration (small volume and clinically silent) is much more common, which can have a frequency of up to $94 \%$ of patients who are in $\mathrm{MV}^{3}$ The consequences of bronchoaspiration depend on chemical composition and volume of the aspirated material, as well as of the presence and virulence of infectious agents and morbid conditions of the patient Figure 1A. Composition of the aspirated material, not only acid or liquid but also non-acid or gaseous, and in addition the presence of pepsin and bile salts, are aggressions for the delicate respiratory epithelium. Bronchoaspiration of such oropharyngeal contents is the main risk factor for pneumonia in intubated and mechanically ventilated patients. ${ }^{4}$

Clinical experience shows that current preventive strategies are insufficient to abolish GER as a risk of ventilatorassociated pneumonia (VAP). It is pertinent, reasonable and mandatory, to search for additional strategies that allow complementing the current conventional measures to reduce the risk of bronchoaspiration in these patients. ${ }^{5}$ In this sense, we have considered that there is both high clinical interest and scientific plausibility regarding an additional practical utility of SUPRAtube, understanding that it is directly related to nosocomial pneumonia risk management. Suction of the oropharyngeal and gastroesophageal reflux (GER) contents that accumulate in the supraglottic sphere represents one of the main risk factors for nosocomial pneumonia, especially in patients under mechanical ventilation. Our impression is that there is a conformism considering that we already do everything necessary to prevent bronchoaspiration and GER in these patients. It is evident that the usual treatment simplified to a prokinetic, oropharyngeal decontamination, semirecumbent body position, gastric acidification, suction of bronchoaspirated secretions, saliva ejector, esophageal balloon probes, among others, are insufficient to prevent nosocomial pneumonia, for which multiple other additional prevention strategies are needed. Antibiotics seem to be few or inadequate in this line because of the risk of multi-resistance selection. In the real-life clinical setting, identifying the causes of GER involves indisputable diagnostic complexity that varies as the patient is under critical care. GER is modifiable in these patients, but therapeutic interventions require the consideration of a comprehensive diagnosis of its causes in order to intervene in a multimodal way and mitigate risk. We consider that applied research should continue evaluating additional innovative strategies such as continuous or intermittent suction
A

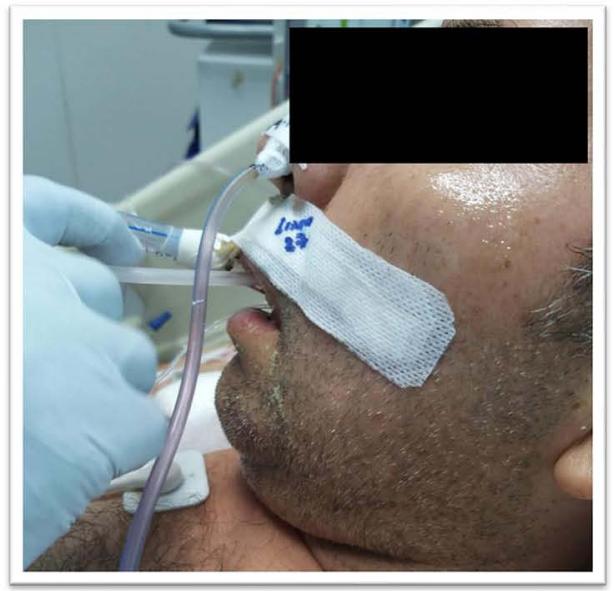

B

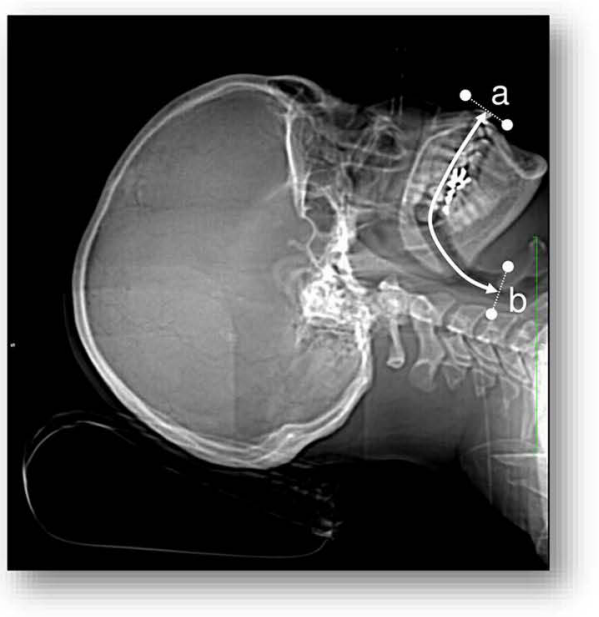

Figure I (A) Example of the usual technique of suction of oropharyngeal content in intubated patients. Representative figure of the most common respiratory therapy technique to aspirate the content of secretions, gastroesophageal reflux and saliva accumulated in the nasopharyngeal territory of intubated and mechanically ventilated patients. The suction is not continuous but intermittent and totally dependent on the health professional. The suction tube in general is semi-rigid. Aspiration is carried out through the connection to a wall vacuum or portable source. The professional therapist must occlude the proximal leak to achieve suction when inserting the probe into the mouth and releasing it to avoid traumatic adhesion to the oropharyngeal tissue. (B) Representative image of the radiological studies of the oropharyngeal area. Representative figure of the CT images with which the fundamental anthropometric studies were started to determine the internal length of the device (defined as the distance from a reference line at the level of the dental arch (A) to a line at the level of the vallecula (B), and the size of the functional end (thimble) of the SUPRAtube device). Multiple anatomical reference relationships were used to define the fundamental morphological characteristics of the device and the location in the nadir zones. The mean values of the reference distances and their dispersion (SD) are described in the body of the document. 
devices, artificial intelligence, augmented reality, application of information and communication technologies (ICTs), among others, to identify new forms of preventive and therapeutic intervention against the risk and consequences of $\mathrm{NN}$ in patients hospitalized in the ICU for various causes.

The objectives of the present study were to determine the ideal shape, size and positioning of a device that allows continuous oropharyngeal suction in mechanically ventilated humans; to evaluate performance of the SUPRAtube supraglottic suction device functional model, in terms of suction capacity (velocity-volume ratio), adequate design, and ideal material(s) to achieve suction, thus avoiding device collapse and adhesion to the oropharynx soft tissues; and to build a definitive prototype of the SUPRAtube, and evaluate its performance in mechanically ventilated patients.

\section{Methods}

Methodological aspects are further described in the repository addendum available on the website (Supplementary Material).

\section{Ethical Considerations}

The study protocol was reviewed and approved by both Institutional Review Board and Ethics committee (Comité de Ética de la Fundación Cardiovascular de Colombia, CEI-FCV); certificate ref. 361, December 2014 for phases 1 and 2; certificate ref. 424, May 2017 for Phase 3. The patient's next of kin have provided informed consent for the images to be published.

\section{Aspects Related to the Study on Swine Heads}

The studies were conducted on swine heads obtained from the meat industry. The swine heads did not imply a biological risk, as they were obtained from meat production of certified animals for human consumption. The heads were cremated following the sanitary protocol of the institution.

\section{Aspects Related to the Study on Humans}

CT scans from intubated and non-intubated patient groups were retrospectively identified from the inventory of brain and neck images obtained from adult individuals for any medical indication and that were available in the institution's radiology department. To be evaluated, the images were always anonymized. Images of cases with craniocervical trauma, apparent deformities or craniocervical surgery were excluded. In addition, 10 intubated and mechanically ventilated patients were included (Table 1). The regulations of the World Medical Association for Research on Humans (Helsinki Declaration) were complied with. Instrumentation was always in accordance with the rules of good clinical practice. Participation in the study included informed and written consent from the close responsible family members of each intubated individual (next of kin). The Written Informed Consent Sheet was signed by the next of kin after reading and knowing the objectives, techniques to be used and any inconvenience that participation could cause. Patient confidentiality was always respected. The study was registered in clinicaltrials.gov (identifier NCT03573609). The present study includes the interim analysis conducted before data collection has been completed for the clinical trial. The authors intend to share with other individuals deidentified.

\section{Project Operational Organization}

For the Development and Scope of the Main Objective and each of the Specific Objectives of the SUPRAtube Project, components and activities were operatively organized into three complementary "Work Phases".

The first phase was focused on "ANTHROPOMETRIC STUDY" in head and neck tomography (50 of nonintubated patients, 50 of intubated patients) (Figure 1B).

The second phase resolved the "DESIGN OF THE SUPRAtube DEVICE AND VALIDATION IN SWINE HEADS". We designed the conceptual (theoretical) model (Figure 2) and evaluated the functional prototype of the device on porcine heads (Figure 3 ). We verified the absence of adhesion to the soft tissue in the anatomical portion. We conducted analysis of suction speeds and volumes. We designed plans, renders and technical reports that demonstrate functionality of the prototype. In a group of functional models, we built and verified operation of the SUPRAtube device, including design of the eventual fixation system to the orotracheal tube; we documented the construction process and the tests carried out, as well as their results. We designed a detailed engineering model of the selected SUPRAtube model with modelling, construction plans, renders, simulation of the designed system, and technical support.

The third phase presented the "EXPLORATORY EVALUATION OF THE SUPRAtube IN PATIENTS". We prospectively identified patients hospitalized in the Intensive Care Unit, with orotracheal intubation, and requiring mechanical ventilation. The effect of SUPRAtube was studied in ten 
Table I General Characteristics of the Study Population

\begin{tabular}{|c|c|c|}
\hline Characteristics & Variables & SUPRAtube \\
\hline Patients, n (\%) & & $10(100)$ \\
\hline Age (years) & Median (min-max) & $58(21-84)$ \\
\hline \multirow[t]{3}{*}{ Age groups, n (\%) } & $<39$ years & $3(30)$ \\
\hline & $40-59$ years & $3(30)$ \\
\hline & 60 and more years & $4(40)$ \\
\hline Sex, n (\%) & Males & $7(70)$ \\
\hline BMI $\left(\mathrm{kg} / \mathrm{m}^{2}\right)$ & Median (min-max) & $24.6(\mid 4.7-51.1)$ \\
\hline Comorbidities, n (\%) & Yes & $7(70)$ \\
\hline Base treatments, n (\%) & Yes & $7(70)$ \\
\hline \multirow[t]{7}{*}{ Vital signs } & $\mathrm{H}_{\mathrm{R}}(\mathrm{bpm})$, mean $(\mathrm{SD})$ & $71.2(19)$ \\
\hline & $\begin{array}{l}R_{R}(r p m) \text {, mean (min- } \\
\max )\end{array}$ & $14(12-19)$ \\
\hline & $\begin{array}{l}\text { Systolic blood pressure } \\
(\mathrm{mmH}) \text {, mean }(\mathrm{SD})\end{array}$ & $132(18)$ \\
\hline & $\begin{array}{l}\text { Diastolic blood pressure } \\
(\mathrm{mmHg}) \text {, mean }(\mathrm{SD})\end{array}$ & $68.4(18.9)$ \\
\hline & $\begin{array}{l}\text { Mean systemic blood } \\
\text { pressure }(\mathrm{mmHg}) \\
\text { median (min-max) }\end{array}$ & $83.3(70.6-111.6)$ \\
\hline & $\begin{array}{l}\text { Body temperature }\left({ }^{\circ} \mathrm{C}\right) \text {, } \\
\text { mean }(\mathrm{SD})\end{array}$ & $36.9(0.5)$ \\
\hline & $\begin{array}{l}\text { Pulse-oximetry (\%), } \\
\text { mean (SD) }\end{array}$ & $93.7(3.4)$ \\
\hline $\begin{array}{l}\text { Antibiotic treatment } \\
\text { prior study period, } \mathrm{n}(\%)\end{array}$ & Yes & I (10) \\
\hline $\begin{array}{l}\text { Antibiotic treatment } \\
\text { during study period, } \\
n(\%)\end{array}$ & Yes & $8(80)$ \\
\hline $\begin{array}{l}\text { Mechanical Ventilation } \\
\text { before study, days ( } n \text { ) }\end{array}$ & & $0.5(0-5)$ \\
\hline \multirow{4}{*}{$\begin{array}{l}\text { Diameter of } \\
\text { Orotracheal tube (F) }\end{array}$} & 7 or less & 0 \\
\hline & 7,5 & $6(60)$ \\
\hline & 8 & $3(30)$ \\
\hline & 8,5 & I (10) \\
\hline \multirow{3}{*}{$\begin{array}{l}\text { Ventilation Mode during } \\
\text { study period }\end{array}$} & AC-VC-plus & $5(50)$ \\
\hline & $\mathrm{AC}-\mathrm{CP}$ & $3(30)$ \\
\hline & $A C-C V$ & $2(20)$ \\
\hline
\end{tabular}

(Continued)
Table I (Continued).

\begin{tabular}{|c|c|c|}
\hline Characteristics & Variables & SUPRAtube \\
\hline \multirow[t]{4}{*}{$\begin{array}{l}\text { Ventilatory Parameters } \\
\text { during study period }\end{array}$} & $\begin{array}{l}V_{\mathrm{T}}(\mathrm{mL}) \text {, median (min- } \\
\max )\end{array}$ & $425(280-680)$ \\
\hline & $\begin{array}{l}V_{E}(1 / m i n) \text {, median (min- } \\
\max )\end{array}$ & $7.1(4.2-9.9)$ \\
\hline & $\mathrm{F}_{1} \mathrm{O}_{2}$, median (min-max) & $0.45(0.3-0.6)$ \\
\hline & $\begin{array}{l}\operatorname{PEEP}\left(\mathrm{cmH}_{2} \mathrm{O}\right) \text {, median } \\
\text { (min-max) }\end{array}$ & $7.5(6-10)$ \\
\hline $\begin{array}{l}\text { Mechanical ventilation } \\
\text { using SUPRAtube device, } \\
\text { hours }(n)\end{array}$ & & $72(7-240)$ \\
\hline $\begin{array}{l}\text { Total aspirate using } \\
\text { SUPRAtube, weight (gr) }\end{array}$ & & $180.5(107-|36|)$ \\
\hline $\begin{array}{l}\text { Suctioned content vs } \\
\text { time ratio }(\mathrm{g} / \mathrm{h})\end{array}$ & & $2,5(15,3-5,70)$ \\
\hline
\end{tabular}

Notes: General descriptive variables of patients included in the pilot study to evaluate the SUPRAtube device in intubated and mechanically ventilated patients. Efficacy and safety variables of the device are also described. The oropharyngeal content was quantified in weight measurements (and not volume) to prevent bubbles from inducing overestimations of the total recovered volume. Abbreviation: IQR, interquartile range.

$(\mathrm{n}=10)$ patients, who received standard treatment throughout the study without their participation in the study implying an omission or a therapeutic addition different from those conventional and required by their medical condition that led to intubation and mechanical ventilation in the Intensive Care Unit. Participation in the study did not change the basic treatment or commodities of any patient. All of them received the usual and necessary medical strategies for the management of their underlying disease that justified the hospitalization in the ICU as well as for eventual complications. Patients were studied in one condition and only once, with the SUPRAtube being inserted by members of the research group. The sample size was defined by consensus and convenience. All patients had a nasogastric tube. During the study, the standard treatment was maintained, without the patients' participation in the study implying an omission or a therapeutic addition different from those conventional and required by their medical condition that led to intubation and mechanical ventilation. The device was inserted buccally, and its positioning was verified using a video bronchoscope for supraglottis evaluations. It was connected to continuous wall suction through conventional tubing with a collecting trap flask inserted in circuit. Suction was maintained continuously during the mechanical ventilation period (Figures 4 and 5). 

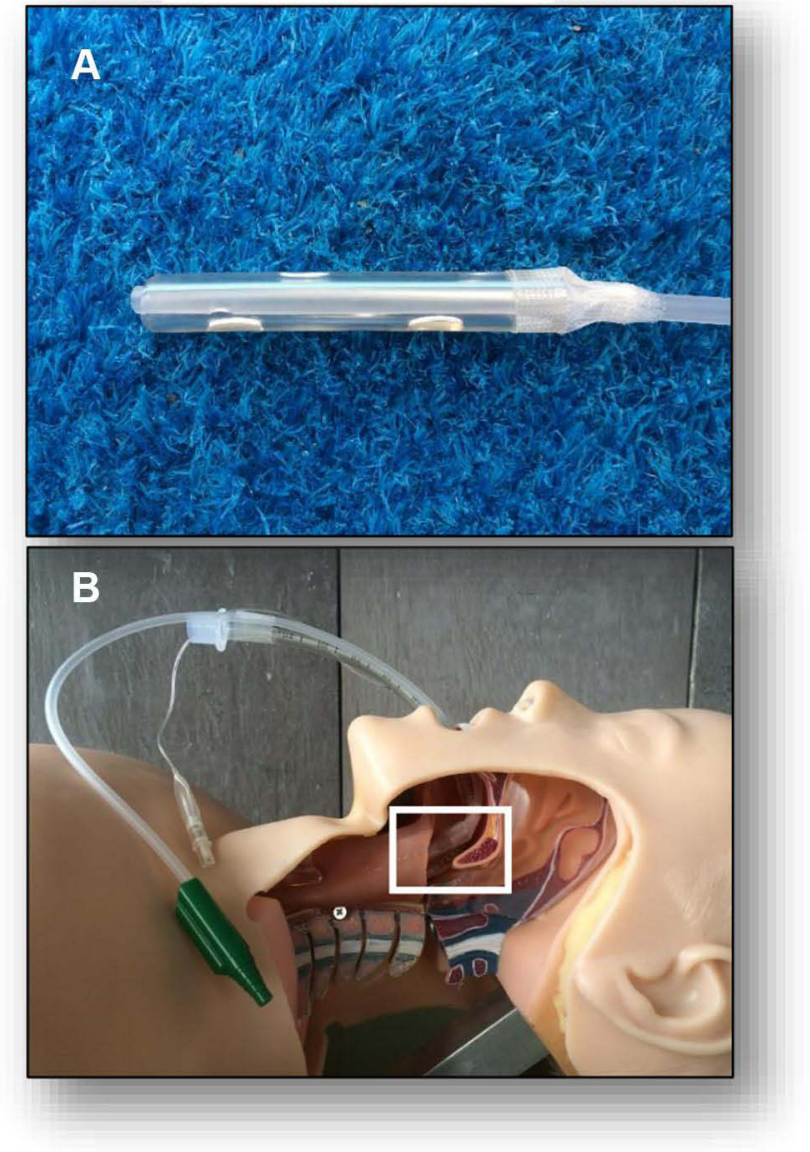

Figure 2 (A) Conceptual model of the SUPRAtube device. (A) shows the conceptual model of the oropharyngeal suction device in terms of the morphological requirements of being tubular, with multiple foramina arranged in several quadrants and having a length proportional to the nadir zone of the oropharynx. The tip of the conceptual model is represented by the white square. This conceptual model was evaluated in life-size human manikins (b) with orotracheal intubation, in order to perform the simulated analysis of the positioning in the nadir area (red rectangle) in mechanically ventilated patients. (B) Representative image of the radiological studies of the oropharyngeal area. Representative figure of the CT images with which the fundamental anthropometric studies were started to determine the internal length of the device (defined as the distance from a reference line at the level of the dental arch (A) to a line at the level of the vallecula (B), and the size of the functional end (thimble) of the SUPRAtube device). Multiple anatomical reference relationships were used to define the fundamental morphological characteristics of the device and the location in the nadir zones. The mean values of the reference distances and their dispersion (SD) are described in the body of the document.

\section{Statistical Analysis of the Results}

Volume of oropharyngeal secretions aspirated per unit of time. We recorded demographic and anthropometric characteristics, patient morbidity, orotracheal tube used, ventilatory modality used, airway pressure, tidal volume, minute volume, respiratory rate, mean pressure, SUPRAtube suction cannula peaks and valleys during continuous oropharyngeal aspiration, volume of oropharyngeal secretions aspirated per unit of time, and complications during device usage (erosions, lacerations, bleeding, displacement, migration, need for removal). As it is the functional design and definitive prototype of a biomedical device, the data obtained relevant to the objectives were those corresponding to the volumes and weights of aspirated secretions, which were analyzed as a dependent variable and in absolute values.

\section{Results \\ Phase I}

The anthropometric study allowed obtaining average measurements of the triangle distances, determined by dental arch, posterior oropharynx and vallecula. We measured the oropharynx diameter in axial projection (Table 1). We identified the nadir decline zone (distance and diameter) as the "target" zone for ideal supraglottic suction. We designed the SUPRAtube device through the Bioengineering Design and Development process (Figure 6A). Several primary conceptual designs were converted into functional models. We tested as many new designs as needed. Evaluation of the various functional models allowed the identification of several fundamental safety challenges, such as: preventing adherence-suction of oropharyngeal tissues; preventing a bloody tip; preventing negative pressure collapse and preventing obstruction of the suction end by secretions and thick fluids. The solutions to challenges made it possible to reach a sufficient and consensual design as an ideal end device, the SUPRAtube. Specifically, multiple foramina sequentially aligned in four quadrants were included in conjunction with a distal apical foramen to prevent adhesion-suction of oropharyngeal tissues (Figure 6B). We developed a suction indirectly distributed on the suction thimble by means of a coaxial tube of smaller diameter that reduces the risk of adhesion. We decided that the distal end of the suction device should be blunt, soft and malleable to make it less bloody. And, to prevent dynamic collapse problems, we designed the distal O-ring, which provides stability against transverse axial forces, maintaining malleability of the end against longitudinal forces. We built device prototypes with all the expected features of safety, reliability, ergonomics and function. The device was based on current quality standards for the manufacture of biomedical equipment.

\section{Phase 2}

Evaluations in porcine models confirmed its correct functioning even in small-diameter spaces, as a representation of the nasal passages and sinuses. 

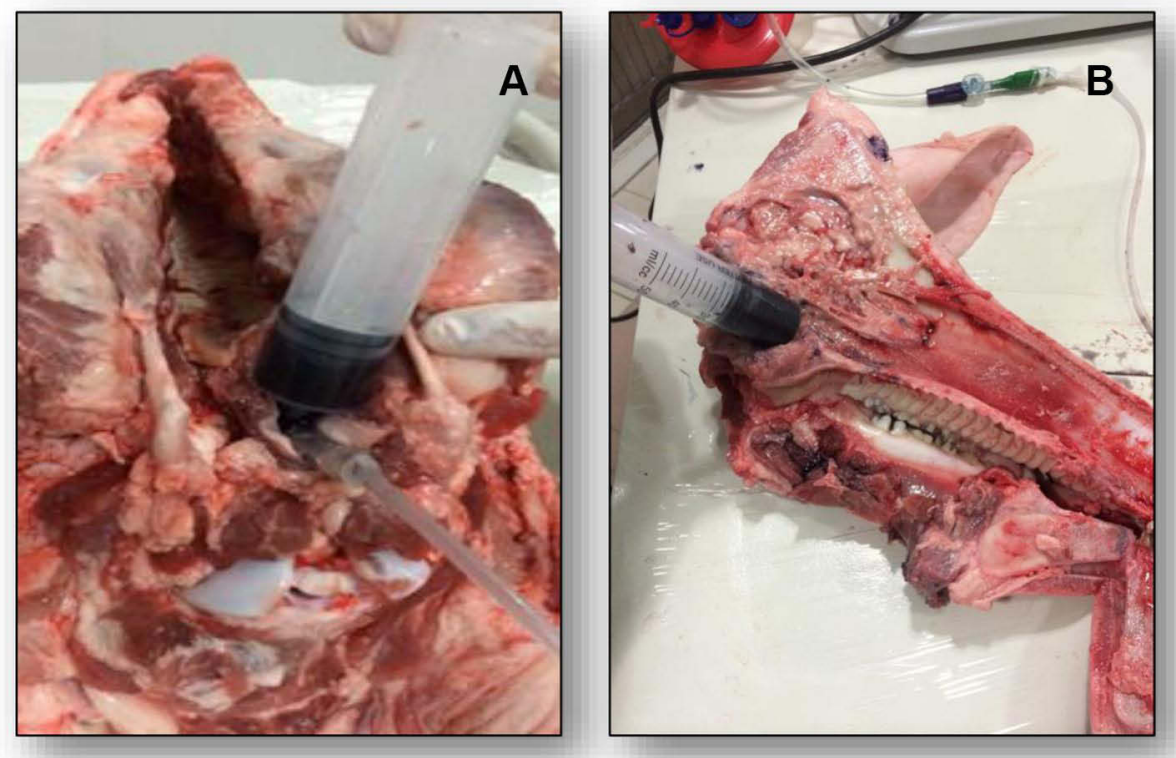

Figure 3 Evaluation of the functional model of the supratube device in animal model ex vivo of porcine HEADS. (A) exemplifies experiments designed to simulate acute high volume fluid accumulation in the oropharyngeal area of porcine heads. It was assumed that this type of experimentation would allow evaluating the mechanical behavior of the device in the face of the imposed vacuum and in intimate contact with rhino-oropharyngeal structures. (A) shows the moment of instillation of liquid marked with methylene blue at the level of the oropharynx, while (B) shows the type of experiments performed on rhinopharynx. For more technical details, please refer to the text.

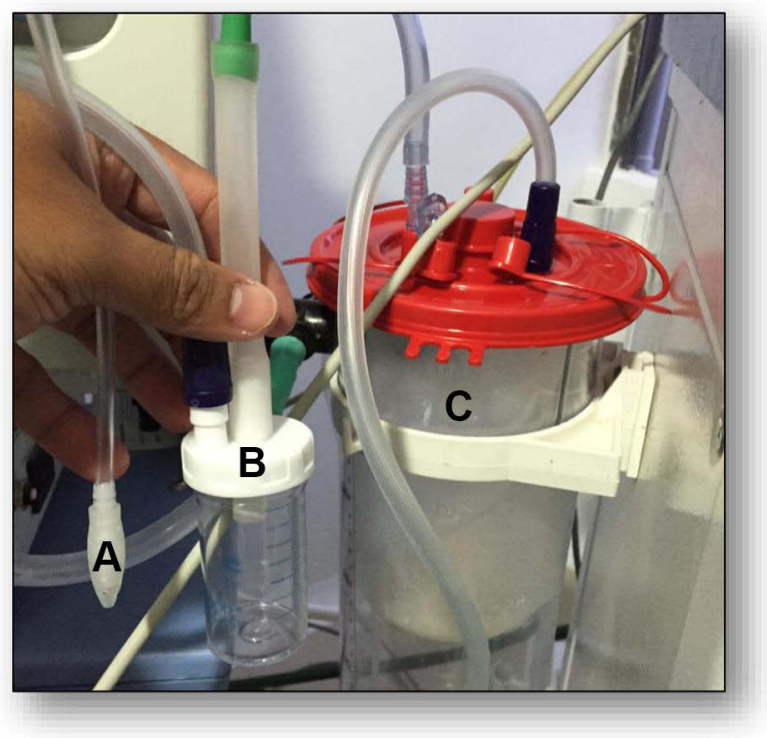

Figure 4 Assembly of wall vacuum, collector vessel, bottle-trap and SUPRAtube. For the different studies, a general assembly was used that included the wall vacuum (VacuTron ${ }^{\circledR}$, not shown), (A) the SUPRAtube device with generic tubing, (B) collecting vessel and (C) a volumetric calibrated trap flask, all of them translucent in order to visualize the liquid content that could eventually be obtained through aspiration once the SUPRAtube started its operation. All the elements were disposable, sterile and complying with all safety standards for patients.

\section{Phase 3}

Sequentially, we proceeded to evaluate functional characteristics in terms of suction capacity in ICU patients, with endotracheal intubation and requiring mechanical ventilation (video available). We performed the second endoscopic study upon study completion, also by video bronchoscopy, emphasizing potential complications due to device pressure, suction, vacuum or contusion. We evaluated complications and the presence of secretions in vallecula, pyriform sinuses, vocal cords or some other areas of the oropharynx, through endoscopic evaluation (Figure 7). Demographic and anthropometric characteristics, and patient morbidity did not demonstrate any connection with device insertion or operation difficulties. The SUPRAtube device did not affect the patients' orotracheal tube at all, nor was it related to the ventilatory modality used, airway pressure, tidal volume, minute volume, or respiratory rate. Mean pressure, peak and valley, in the SUPRAtube suction cannula always remained stable between values of -20 to $-30 \mathrm{mmHg}$. Measurement of the aspirated volume was made difficult by the presence of foam, for which weight was assumed as the objective variable less susceptible to measurement bias. One of the most striking points is that the initial oropharyngeal suction volume in some cases reached up to $415 \mathrm{~mL}$ in only approximately 6 hours, despite the fact that patients were receiving standardized conventional respiratory therapy strategies (Figure 8). The weight of oropharyngeal secretions aspirated per unit of time varied widely among 


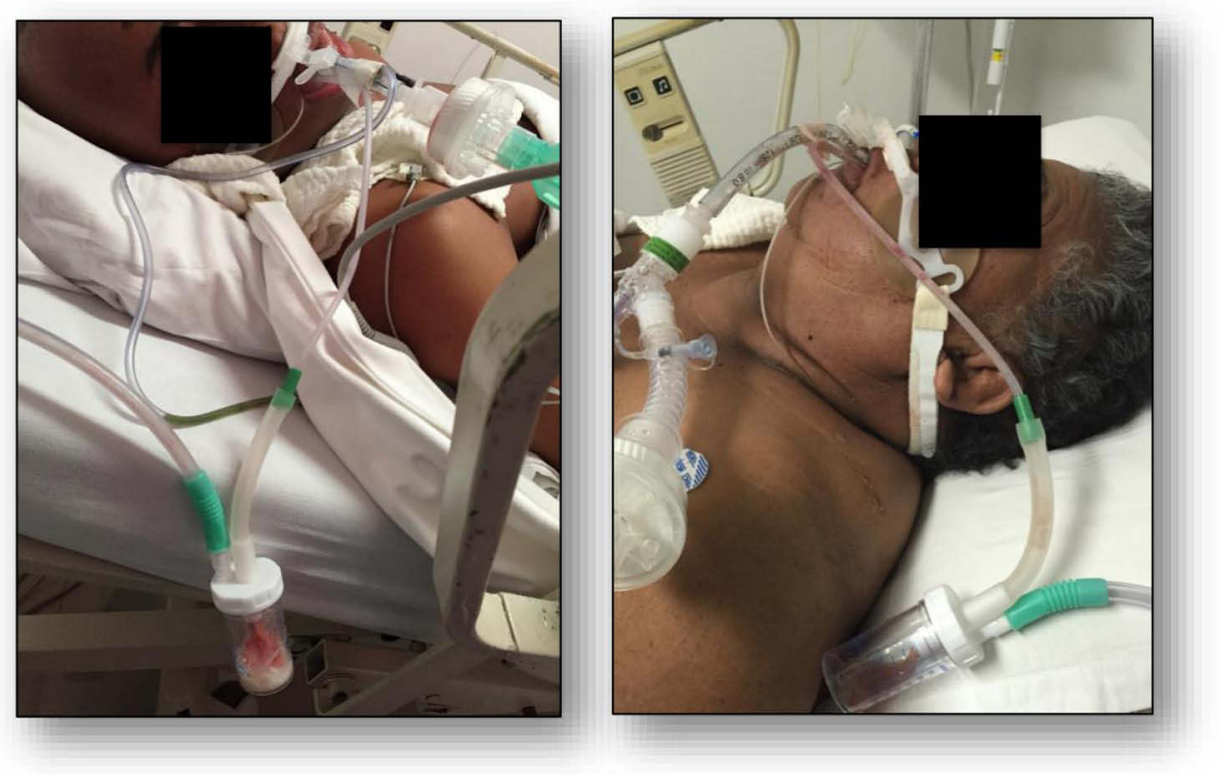

Figure 5 Assembly of wall vacuum, collector vessel, bottle-trap and SUPRAtube. Representative figure of the SUPRAtube continuous suction system in the assembly form and in full operation in two patients, in which the volume of oropharyngeal content that was obtained acutely (flask-trap) at the time of its insertion is clearly evidenced and start of suction, even with the patient in a semi-recumbent position.

A

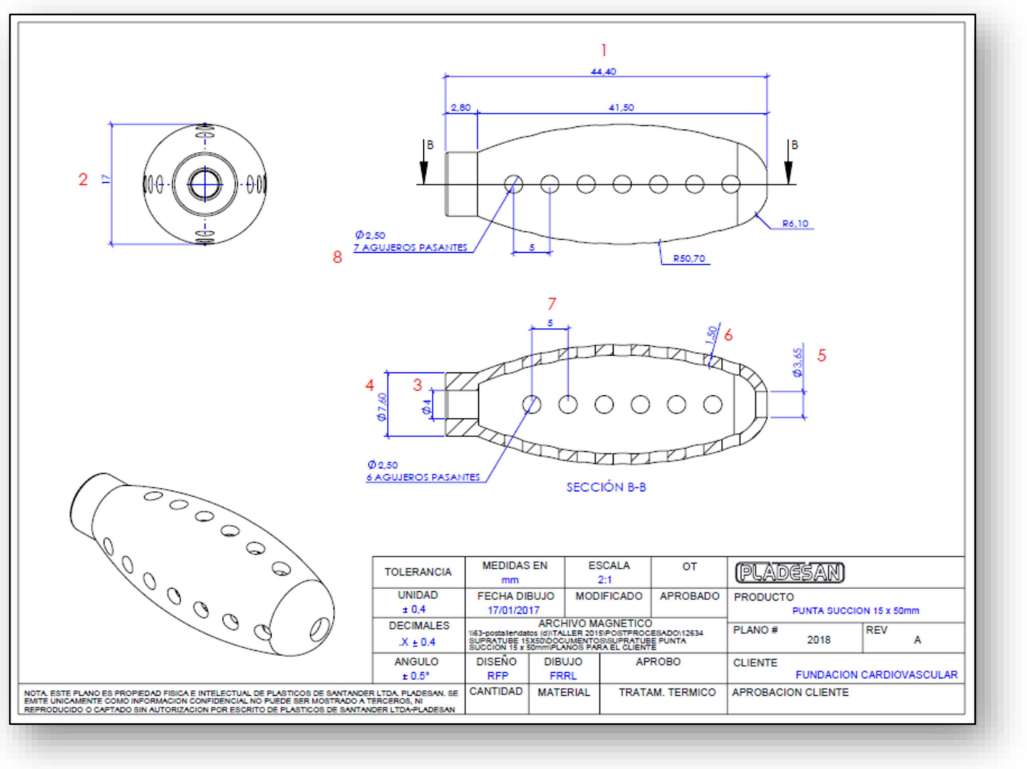

B
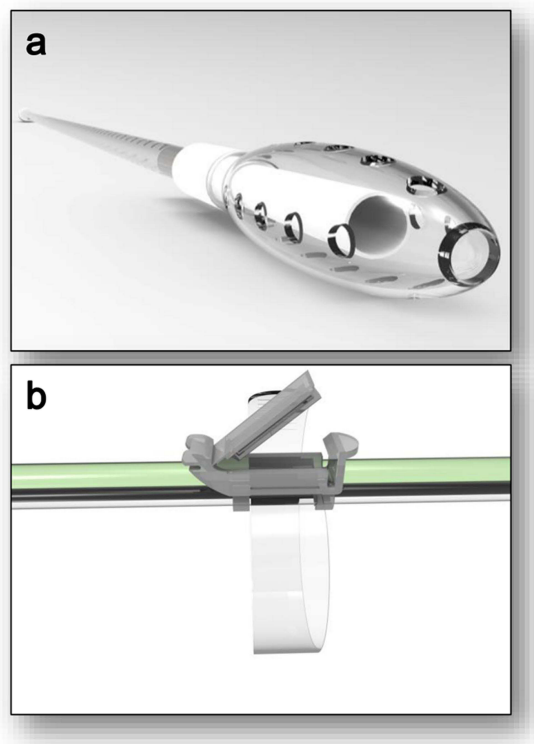

Figure 6 (A) Representative figure of the industrial design drawings of the SUPRAtube. The intention of taking the SUPRAtube device to the molding, injection, clinical validation and future commercialization process has required all the necessary plans being designed in accordance with current regulations. These design drawings are also necessary to file the patent application. For more details on the respective plans, please contact the principal investigator. (B) Specialized 3D graphic design of the end of the SUPRAtube and the fastening system to the orotracheal tube. The SUPRAtube, at its distal end which is inserted into the oropharynx, has the appearance shown in (a). The number of foramina in opposite quadrants and at the end of the device is clearly observed, a design that was validated as the most appropriate to allow high suction capacity but minimal risk of secondary adhesion and trauma. (b) Shows the fastening system designed to fix the SUPRAtube to the orotracheal tube, with ease of releasing and repositioning it by means of an opening and closing tab. 


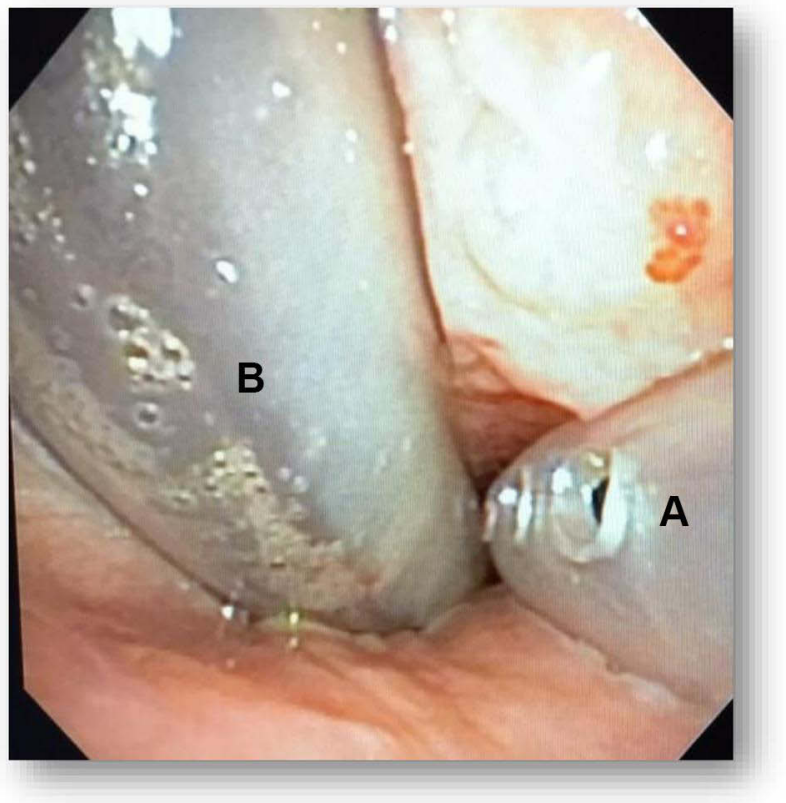

Figure 7 Videoendoscopic view of supraglottic anatomic area using fiber endoscopy in a patient with orotracheal tube and supratube. Representative figure of the type of endoscopic vision that allowed evaluating the positioning of the SUPRAtube end (A) in patients with orotracheal tube (B) and mechanical ventilation. In the case, it can be seen how the medial SUPRAtube foramina remain permeable and functional for aspiration in the event that respiratory secretions, gastric content or saliva accumulate.

volunteers. Higher values were quantified within the first hour $(65 \pm 110 \mathrm{gr})$ and decreased in the following hours. No erosions, lacerations, bleeding, displacement, migration, or need for removal were observed in any case due to adverse events related to the device (Table 2).

\section{Discussion}

The present study describes an original technological development motivated by a real need perceived within our clinical experience: the potential accumulation of naso-oropharyngeal secretions and gastroesophageal reflux products in the oropharynx area of patients who are admitted to the ICU with mechanical ventilation. These secretions represent a widely recognized risk factor for the development of health care-associated pneumonia and other respiratory complications. ${ }^{6}$ It is reasonable to try that the content that accumulates in the oropharynx does not pass through the glottis, as an attempt to reduce colonization and infection of the lower respiratory tract. ${ }^{7}$ This oropharyngeal content is the result of an eventual combination of saliva, sinus secretions, nasal secretions, bleeding and gastroesophageal reflux. We have hypothesized that a pre-bronchoaspiration suction system could have a beneficial impact in addition to the multiple usual strategies for the prevention of bronchoaspiration of oropharyngeal content of any origin and, consequently, could reduce the risk of tracheobronchitis, pneumonia and other complications associated with health care. Several aspects must be highlighted:

The first aspect is the original nature of the study. As far as we have been able to find, there is no specific device for continuous suction of oropharyngeal (supraglottic) content in intubated and mechanically ventilated patients. Our device had to be conceptualized, designed, prototyped and validated without being able to compare it with another device with similar characteristics. This becomes a strength of the study itself and determines that the SUPRAtube device has been patented as a utility model.

The second aspect is relevance of the SUPRAtube device. Our results suggest that the fluid accumulated in the supraglottis is frequent and abundant, and often underestimated. In this sense, it is suggested that saliva production can reach up to $800-1000 \mathrm{~mL} / 24$ hours in healthy individuals. If they are intubated, the accumulation of liquid content in supraglottis is even greater due to presence of the orotracheal (or nasotracheal) tube, supine body position, decreased abdominal compliance, gastric distension, compression of the esophagus by the pneumotracheal balloon, alteration of swallowing dynamics or absence of swallowing reflex, muscle relaxant drugs, sedation, and presence of nasogastric or orogastric tube, among others.

The third aspect is clinical relevance of the aspiration of contents accumulated in supraglottis. It has been suggested that $45 \%$ of healthy individuals habitually aspirate during sleep. Commonly, hospitalized patients are colonized by microorganisms acquired from hospital environment, and up to $75 \%$ of seriously ill patients get colonized within $48 \mathrm{~h} .{ }^{8}$ The first route of lung infection is precisely through microaspiration of microorganisms colonizing the oropharyngeal secretions. The main complication of bronchoaspiration is infection of the respiratory system, with bacterial tracheobronchitis and hospital-acquired pneumonia (HAP) being the most frequent. ${ }^{9}$ HAP leads to the causes of hospital-acquired infection deaths with a mortality of between 20 $50 \%{ }^{10-12}$ and, far from being an eradicated problem, it affects a high percentage of patients admitted to any hospital both in Colombia and outside the country. Ventilatorassociated pneumonia (VAP) is the most frequent nosocomial infection in critically ill patients and is one of the main causes of prolonged hospitalization and ICU mortality. 


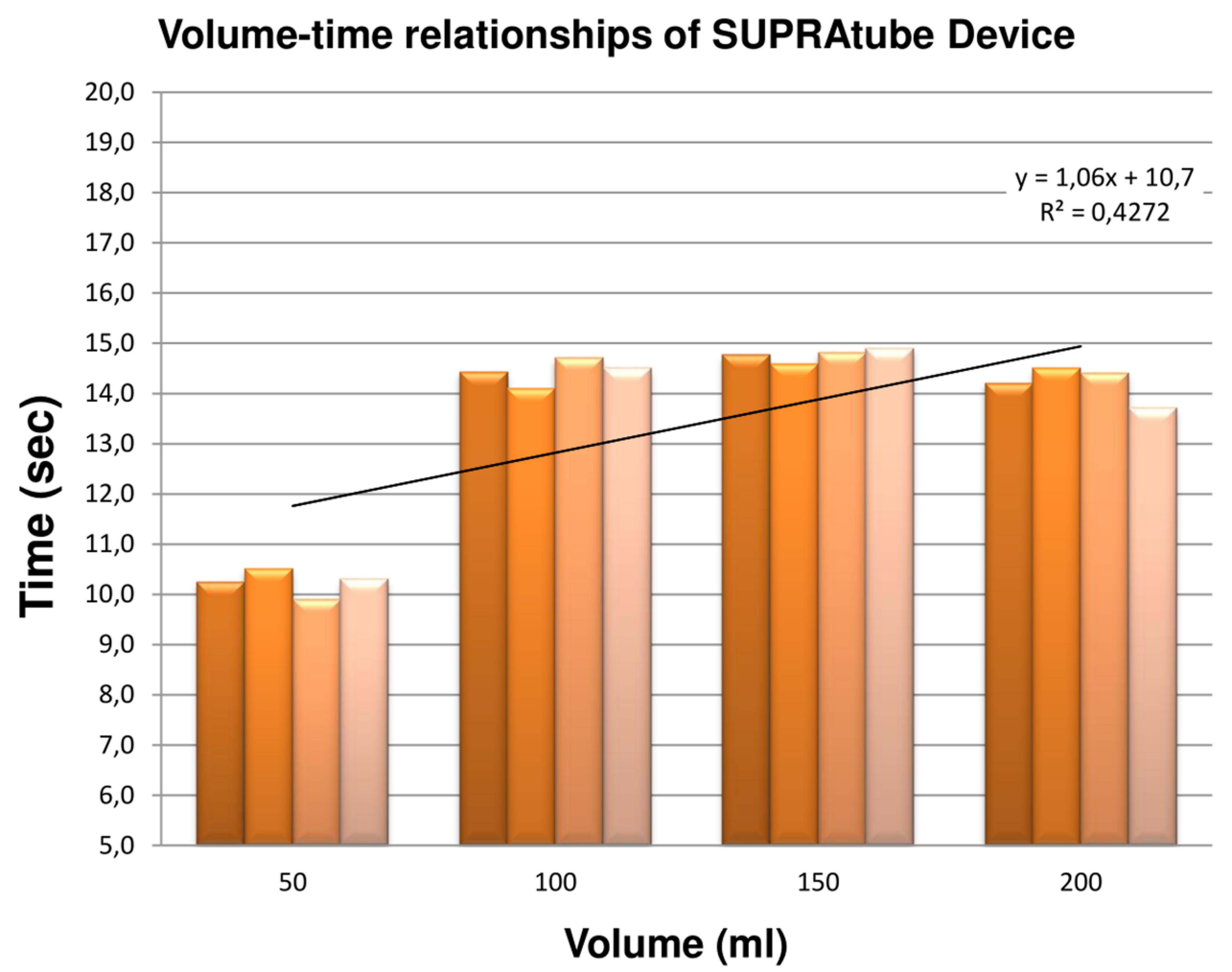

Figure 8 Evaluation of the volume-time relationships of suctioning using the supratube device in laboratory conditions. One of the most attractive points of the SUPRAtube design is its high suction capacity, denoted in this figure as the two-dimensional ratio of the aspirated volume (abscissa, in $\mathrm{mL}$ ) per unit of time (ordinate, in sec.). This type of approach allowed us to emulate the most dramatic conditions of fluid accumulation in the mouth, as can occur in cases of emesis, epistaxis or upper gastrointestinal bleeding. These evidences allowed us to consider the current SUPRAtube design valid and efficient. For more details, please refer to the text.

Severity depends on the number and virulence of pathogens entering the lower respiratory tract. ${ }^{13}$ Bronchoaspirationassociated lung injury is characterized by lung inflammation,

Table 2 Fiberendoscopic Assessment of Supraglottic Adverse Events in Patients Submitted to Supratube Treatment: Baseline vs Final Evaluations

\begin{tabular}{|l|c|c|}
\hline Fiberendoscopic Evaluation of Supraglottis & Initial & Final \\
\hline Local edema & 4 & I \\
\hline Local eritema & 0 & 0 \\
\hline Local fibrinosis & 0 & 0 \\
\hline Regional lacerations & 3 & 2 \\
\hline Local necrosis & 0 & 0 \\
\hline Oropharyngeal bleeding & I & 0 \\
\hline Needing of removal of the device for any cause & 0 & 0 \\
\hline
\end{tabular}

Notes: Findings of fiberendoscopic assessment of supraglottic integrity prior to (initial) and following (final) the SUPRAtube use in two study evaluation points. Data represent absolute values of patients ( $n$ ) disclosing the adverse event. The study group assign per consensus the potential cause-effect relationship of the findings with the use of the SUPRAtube device or other events related with orotrachea tube, nasogastric tube or other procedures. No relationship were identified between SUPRAtube and adverse events. capillary leakage, and oxidative damage. Possible outcomes range from mild pneumonitis to acute respiratory distress and death. The incidence of VAP ranges between $10 \%$ and $20 \%$ of patients with more than 48 hours on mechanical ventilation, and the attributable mortality doubles that of patients not suffering from it. ${ }^{14-16}$

The fourth aspect to highlight has to do with technical features of the SUPRAtube device. The materials of the different parts are biocompatible. Device usage is intuitive, and its placement technique is simple. The fixation system is universal regardless of the type of endotracheal tube used. The suction system connections have been designed so that they have connectivity with conventional tubes. The possibility of interspersing a collecting vessel allows continuous quantification of the volume of oropharyngeal content aspirated, a variable that to date has not been controlled or identified in the conventional clinical settings in which we have considered use of the device. The tube is translucent, which makes it possible to evaluate the characteristics of aspirated material. The novel design appears to guarantee that the device aspirates without the possibility of creating a local vacuum in front of the oropharyngeal epithelium. The aspiration capacity (a few seconds 
even for volumes greater than $200 \mathrm{~mL}$ ) allows us to assume that the device will be useful and especially indicated in cases of emesis.

The fifth aspect to highlight is related to device production with polyvinyl chloride (PVC). Life cycle analyses show that the environmental impact of PVC is equivalent to or even more favorable than other materials. The application of existing technology makes PVC one of the most respectful materials with human beings and the environment, being very difficult to replace in some medical devices.

Another relevant aspect is the potential clinical impact of SUPRAtube in preventive terms of tracheobronchitis and pneumonia, associated both with mechanical ventilation and other pathologies or interventions that lead to increased oropharyngeal content. Given that low production price and functional concept of the device provide a possibility for its globalization, future clinical trials may be representing reallife studies in the prevention of morbidity and mortality associated with accumulation of biological fluids from gastroesophageal reflux and the inevitable oropharyngeal colonization in instrumentalized patients.

The present study has limitations that deserve to be mentioned. A potential limitation, intrinsic to future clinical trial in humans, is the impossibility of predefining which patients will present vomiting, macro-regurgitations, sinus secretions or oropharyngeal secretions of greater magnitude. We have not evaluated the possibility of using the SUPRAtube in pediatric patients. There are no data regarding safety of the device operation for several consecutive days. Although we promote its insertion by buccal route, we must keep in mind the possible alternative of nasal introduction. Finally, we did not evaluate the complementary benefits of intermittently instilling some antiseptic solutions through the SUPRAtube itself to reduce risks related to bacterial biofilm and reduce oropharyngeal inoculum. We are convinced that the SUPRAtube device should be evaluated in clinical trials to determine its impact in terms of safety and clinical efficacy in preventing VAP.

The present study allows concluding that the SUPRAtube device is effective and safe to be used in continuous supraglottic suction for intubated and mechanically ventilated patients. SUPRAtube is practical and shows a low production cost. The device appears to be beneficial as a preventive non-redundant strategy that does not exert antibiotic pressure and is complementary to the standard management of intubated and mechanically ventilated patients. These results justify the design of studies to evaluate the efficacy and safety of device operation during all days of mechanical ventilation.

\section{Data Sharing Statement}

The authors intend to share with other individuals deidentified participant data including demographics, clinical characteristics, and specific data regarding safety and efficacy of the device. Data are available on request from the corresponding author (mauricioorozco@fcv.org).

\section{Acknowledgments}

We thank to Nidya López for her assistance in acquisition of data. This study was financed in part by MINCIENCIAS (COLCIENCIAS) Ref. 656677758334 contrato No. 8332017, and Fundación Cardiovascular de Colombia.

\section{Disclosure}

The authors ARS and MOL declare to be inventors with intellectual property of the device. The patrimonial property of the same belongs to the FCV institution. ARS reports grants from MINCIENCIAS, during the conduct of the study, and has a patent Patente Modelo de Utilidad licensed to ref NC2016/0002059. MOL reports grants from Ministerio de Ciencia y Tecnologia de Colombia MINCIENCIAS, during the conduct of the study, and has a patent Patente de modelo de utilidad licensed to ref NC2016/0002059. The authors report no other potential conflicts of interest for this work.

\section{References}

1. Orozco-Levi M, Félez M, Martínez J, et al. Gastroesophageal reflux in mechanically ventilated patients: effects of an oesophageal balloon. Eur Respir J. 2003;22:348-353. doi:10.1183/09031936.03.00048902

2. Schallom M, Orr J, Metheny M, Pierce J. Gastroesophageal reflux in critically ill patients. Dimens Crit Care Nurs. 2013;32:69-77. doi:10.1097/DCC.0b013e318280836b

3. Nseir S, Zerimech F, Jaillette E, Artru F, Balduyck M. Microaspiration in intubated critically ill patients: diagnosis and prevention. Infect Disord Drug Targets. 2011;11:413-423. doi: $10.2174 / 187152611796504827$

4. Koulenti D, Tsigou E, Rello J. Nosocomial pneumonia in 27 ICUs in Europe: perspectives from the EU-VAP/CAP study. Eur J Clin Microbiol Infect Dis. 2017;36:1999-2006. doi:10.1007/s10096-016-2703-z

5. Ferrer M, Bassi GL, Torres A. Medidas prácticas para la prevención de la neumonía nosocomial [Practical measures for the prevention of nosocomial pneumonia]. Medicina respiratoria. 2013;6:33-44. Spanish.

6. Messika J, La Combe B, Ricard J. Oropharyngeal colonization: epidemiology, treatment and ventilator-associated pneumonia prevention. Ann Transl Med. 2018;6(21):426-427. doi:10.21037/atm.2018.10.17

7. Dezfulian C, Shojania K, Collard HR, Kim HM, Matthay MA, Saint S. Subglottic secretion drainage for preventing ventilator-associated pneumonia: a meta-analysis. Am $J$ Med. 2005;118:11-18. doi:10.1016/j.amjmed.2004.07.051 
8. Venditti M, Falcone M, Corrao S, Licata G, Serra P. Outcomes of patients hospitalized with community-acquired, health care-associated, and hospital-acquired pneumonia. Ann Intern Med. 2009;150:19. doi:10.7326/0003-4819-150-1-200901060-00005

9. Yang CS, Qiu HB, Zhu YP, Huang YZ, Xu XT. Effect of continuous aspiration of subglottic secretions on the prevention of ventilator associated pneumonia in mechanically ventilated patients: prospective, randomized, controlled clinical trial. Zhonghua Nei Ke Za Zhi. 2008; $47: 625-629$.

10. Craven DE, Palladino R, McQuillen DP. Healthcare-associated pneumonia in adults: management principles to improve outcomes. Infect Dis Clin North Am. 2004;18:939. doi:10.1016/j.idc.2004.08.001

11. Blanquer J, Aspa J, Anzueto A, et al. Sociedad Española de Neumología y Cirugía Torácica. SEPAR Guidelines for Nosocomial Pneumonia. Arch Bronconeumol. 2011;47:510-520. doi:10.1016/j. arbres.2011.05.013

12. Torres A, Niederman MS, Chastre J, et al. International ERS/ESICM/ ESCMID/ALAT guidelines for the management of hospital-acquired pneumonia and ventilator-associated pneumonia: guidelines for the management of hospital-acquired pneumonia (HAP)/ventilator-associated pneumonia (VAP) of the European Respiratory Society (ERS), European Society of Intensive Care Medicine (ESICM), European Society of Clinical Microbiology and Infectious Diseases (ESCMID) and Asociacion Latinoamericana del Torax (ALAT). Eur Respir J. 2017;50:1700582. doi:10.1183/13993003.00582-2017
13. Dezfulian C, Shojania K, Collard HR, Kim HM, Matthay MA, Saint S. Subglottic secretion drainage for preventing ventilator-associated pneumonia: a meta-analysis. Am J Med. 2005;118:11-18. doi:10.1016/j.amjmed.2004.07.051

14. Pace F, Bianchi Porro G. Gastroesophageal reflux disease: a typical spectrum disease a new conceptual framework is not needed. Am $J$ Gastroenterol. 2004;99(5):946-949. doi:10.1111/j.1572-0241.2004.0 4164.x

15. Nind G, Chen WH, Protheroe R, et al. Mechanisms of gastroesophageal reflux in critically ill mechanically ventilated patients. Gastroenterology. 2005;128(3):600-606. doi:10.1053/j.gastro.2004.12.034

16. Kollef MH, Morrow LE, Niederman MS, et al. Clinical characteristics and treatment patterns among patients with ventilator-associated pneumonia. Chest. 2006;129(5):1210. doi:10.1378/chest.129.5.1210
Medical Devices: Evidence and Research

\section{Publish your work in this journal}

Medical Devices: Evidence and Research is an international, peerreviewed, open access journal that focuses on the evidence, technology, research, and expert opinion supporting the use and application of medical devices in the diagnosis, monitoring, treatment and management of clinical conditions and physiological processes. The identification of novel devices and optimal use of existing devices

\section{Dovepress}

which will lead to improved clinical outcomes and more effective patient management and safety is a key feature of the journal. The manuscript management system is completely online and includes a very quick and fair peer-review system. Visit http:// www.dovepress.com/testimonials.php to read real quotes from published authors. 